\title{
ESTUDO DE UMA COLUNA DE ABSORÇÃO DE DIÓXIDO DE CARBONO EM SOLUÇÃO DE HIDRÓXIDO DE SÓDIO
}

\author{
I. C. P. COUTO ${ }^{1}$, S. C. P. COUTO ${ }^{1}$ e E. N. de OLIVEIRA Jr ${ }^{2}$ \\ ${ }^{1}$ Universidade Federal de São João Del Rei, Departamento de Engenharia Química e Estatística \\ ${ }^{2}$ Universidade Federal de São João Del Rei, Departamento de Química, Biotecnologia e Engenharia \\ de Bioprocessos \\ E-mail para contato: eniobio@ufsj.edu.br
}

\begin{abstract}
RESUMO - $\mathrm{O}$ dióxido de carbono $\left(\mathrm{CO}_{2}\right)$ é um problema nas questões industriais (qualidade dos produtos gasosos, envenenamento de catalisador) e ambientais (efeito estufa). Por isso ele é foco de estudo neste trabalho que visa estudar e promover a absorção deste gás em solução de hidróxido de sódio $(\mathrm{NaOH})$. Utilizou-se uma coluna de absorção laboratorial com conformação que promove o contato em contra corrente do gás $\left(\mathrm{CO}_{2}\right.$ e ar) e o NaOH além de possibilitar a variação da vazão de entrada de $\mathrm{CO}_{2}\left(20,10\right.$ e $\left.5 \mathrm{~L} \mathrm{~min}^{-1}\right)$. Os resultados mostram que quanto maior a vazão de $\mathrm{CO}_{2}$ mais rápido se atinge a saturação do $\mathrm{NaOH}$. Em relação ao $\mathrm{NaOH}$ a absorção fica em torno de $50 \%$ para as três condições estudadas. Em relação à fase gasosa a porcentagem de recuperação máxima de $\mathrm{CO}_{2}$ é $73 \%$ e ocorre na condição de menor vazão de $\mathrm{CO}_{2}$, para esta mesma vazão o coeficiente de transferência de massa, também é máximo, 0,08690 $\mathrm{mol} \mathrm{min}^{-1} \mathrm{~m}^{-3}$. Isto é, estes parâmetros são inversamente proporcionais às vazões de $\mathrm{CO}_{2}$ adotadas.
\end{abstract}

\section{INTRODUÇÃO}

A remoção de dióxido de carbono $\left(\mathrm{CO}_{2}\right)$ tem sido há décadas, um passo essencial em muitos processos industriais de transformação operações, como a síntese de amônia, gás natural, purificação e refino de petróleo. Nestes processos, o $\mathrm{CO}_{2}$ é considerado uma impureza que tem de ser removido a partir de gases industriais, a fim de melhorar a qualidade dos produtos gasosos ou a evitar problemas no processo como envenenamento do catalisador. Nos últimos anos, a remoção de $\mathrm{CO}_{2}$ a partir de fluxos de gases industriais tornou-se ainda mais importante. Isso é resultado da preocupação com meio ambiente e a redução das emissões de gases de efeito estufa a partir de fontes industriais. $\mathrm{O} \mathrm{CO}_{2}$ é considerado o maior contribuinte para o problema do aquecimento global, e é, portanto, o principal objetivo de redução. A remoção de $\mathrm{CO}_{2}$ de correntes de gás pode ser conseguida por absorção em um solvente líquido. Este processo de separação geralmente ocorre nas colunas de absorção recheadas (Aroonwilas et al., 2003).

Os recheios são materiais que promovem o contato direto entre a fase líquida e a fase de gás. Eles proporcionam a transferência de massa eficiente com baixo consumo de energia que são requisitos necessários para a economia industrial (Aroonwilas et al., 2003). 
O presente trabalho visa compreender o processo de absorção quando se utiliza hidróxido de sódio como absorvente de dióxido de carbono.

\section{MATERIAIS E MÉTODOS}

\subsection{Reagentes e Equipamentos}

Os reagentes utilizados foram: água destilada, hidróxido de sódio da Ecibra, ácido clorídrico da Labsynth, Carbonato de sódio Labsynth, fenolftaleína da Impex.

Os equipamentos usados: balança analítica digital Elgin DP 15 plus, cronômetro digital Caston, altímetro e barômetro digital da Oregon, coluna de absorção de $\mathrm{CO}_{2}$ do Laboratório de Engenharia Química da Universidade Federal de São João Del Rei, Campus Alto Paraopeba.

\subsection{Métodos}

Foram preparadas soluções de hidróxido de sódio $0,1 \mathrm{~mol} \mathrm{~L}^{-1}$ e de ácido clorídrico 0,168 mol L-1 (padronizada).

Para cada experimento realizado foi adicionado ao kit de coluna de absorção 14 litros de hidróxido de sódio colorido com algumas gotas de fenolftaleína. Realizaram-se três experimentos com a coluna ligada em condições constantes de vazão de hidróxido de sódio (2 L $\min ^{-1}$ ) e de ar (40 SCIH (normal pé cúbico por hora)). Houve variação na alimentação de $\mathrm{CO}_{2}$ na coluna: $20 \mathrm{~L} \mathrm{~min}^{-1}$; $10 \mathrm{~L} \mathrm{~min}^{-1} ; 5 \mathrm{~L} \mathrm{~min}^{-1}$. Anotou-se a temperatura e pressão ambiente e temperaturas de entrada e saída do gás e da solução de $\mathrm{NaOH}$ durante todos os experimentos. Nos primeiros 2 minutos e meios de experimento a solução de hidróxido foi coletada em um segundo compartimento do equipamento para não recircular na coluna, a fim de medir a absorção de dióxido de carbono sem reciclo.

Para cada experimento retirou-se alíquotas de $10 \mathrm{~mL}$ de hidróxido de sódio nos primeiros 2 minutos e meio e depois a cada cinco minutos para que fosse titulada (em duplicata) com ácido clorídrico a fim de determinar quanto de $\mathrm{CO}_{2}$ estava sendo absorvido pela solução. $\mathrm{O}$ experimento terminava quando a solução de hidróxido de sódio se tornava incolor na coluna e o volume de ácido gasto na titulação fosse baixo.

Para determinar a concentração de $\mathrm{CO}_{2}$ é necessário conhecer as relações estequiométricas das reações. De acordo com a concentração de ácido clorídrico e o seu volume gasto na titulação é possível determinar o número de mols e a massa de $\mathrm{NaOH}$ livre, ou seja, aquele hidróxido que não absorveu $\mathrm{CO}_{2}$. A partir da diferença de mols de $\mathrm{NaOH}$ inicial (no ponto zero) com o número de mol de $\mathrm{NaOH}$ de cada tempo pode-se quantificar quanto de $\mathrm{CO}_{2}$ que foi absorvido na solução de $\mathrm{NaOH}$, tendo portanto a concentração de $\mathrm{CO}_{2}$ absorvida.

\section{RESULTADOS E DISCUSSÃO}

Nos primeiros dois minutos e meio e depois a cada cinco minutos foram aferidos os 
valores das temperaturas de entrada e saída do gás, temperatura de entrada e saída da solução de $\mathrm{NaOH}$, pressão e temperatura ambiente, que foram constantes para todos os pontos, além do volume de $\mathrm{HCl}$ gastos nas titulações. A Tabela 1 apresenta as condições dos três experimentos realizados.

Tabela 1 - Dados experimentais

\begin{tabular}{|c|c|c|c|}
\hline Dados & $1^{\text {a }}$ Condição & $2^{\text {a }}$ Condição & $3^{\text {a }}$ Condição \\
\hline Vazão de ar & $18,88 \mathrm{~L} \mathrm{~min}^{-1}$ & $18,88 \mathrm{~L} \mathrm{~min}^{-1}$ & $18,88 \mathrm{~L} \mathrm{~min}^{-1}$ \\
\hline Vazão de $\mathrm{CO}_{2}$ & $20 \mathrm{~L} \mathrm{~min}^{-1}$ & $10 \mathrm{~L} \mathrm{~min}^{-1}$ & $5 \mathrm{~L} \mathrm{~min}^{-1}$ \\
\hline Vazão NaOH & $2 \mathrm{~L} \mathrm{~min}^{-1}$ & $2 \mathrm{~L} \mathrm{~min}^{-1}$ & $2 \mathrm{~L} \mathrm{~min}^{-1}$ \\
\hline Pressão amb. & $895,5 \mathrm{hPa}$ & $889,8 \mathrm{hPa}$ & $887,9 \mathrm{hPa}$ \\
\hline Temp. amb. & $27,2^{\circ} \mathrm{C}$ & $24,1^{\circ} \mathrm{C}$ & $26^{\circ} \mathrm{C}$ \\
\hline Temperatura da água na entrada & $27^{\circ} \mathrm{C}$ & $23^{\circ} \mathrm{C}$ & $26^{\circ} \mathrm{C}$ \\
\hline Temperatura da água na saída & $26^{\circ} \mathrm{C}$ & $22^{\circ} \mathrm{C}$ & $24^{\circ} \mathrm{C}$ \\
\hline Temperatura do ar na entrada & $26^{\circ} \mathrm{C}$ & $23^{\circ} \mathrm{C}$ & $25^{\circ} \mathrm{C}$ \\
\hline Temperatura do ar na saída & $26^{\circ} \mathrm{C}$ & $23^{\circ} \mathrm{C}$ & $25^{\circ} \mathrm{C}$ \\
\hline
\end{tabular}

O volume de hidróxido de sódio consumido no primeiro ponto foram os mais altos de cada duplicada do experimento ( $1^{\text {a }}$ condição: 8,0 e 7,4 mL; $2^{\text {a }}$ condição: 8,6 e 7,8 mL; $3^{\text {a }}$ condição: 8,2 e $8,8 \mathrm{~mL}$ ). Este volume foi reduzindo no decorrer do experimento até se atingir $0,2 \mathrm{~mL}$ de hidróxido no último ponto de cada condição.

A primeira análise que se faz do experimento é que absorção de $\mathrm{CO}_{2}$ no hidróxido de sódio é uma reação visível devido à perda da coloração rósea do absorvente. A temperatura de entrada do ar é menor que a temperatura ambiente porque o gás encontra-se comprimido e a sua expansão gera decaimento da temperatura.

Com o volume de $\mathrm{HCl}$ necessário para titular a solução de $\mathrm{NaOH}$ foi possível calcular o números de mols e a concentração de $\mathrm{CO}_{2}$ absorvidos em $10 \mathrm{~mL}$ da solução de hidróxido de sódio. Os valores das concentrações de dióxido de carbono ( C CO2) e das concentrações de hidróxido de sódio ( $\mathrm{C} \mathrm{NaOH}$ ) são as médias das duplicatas realizadas no experimento.

Há uma diferença na concentração inicial de $\mathrm{NaOH}$ nas três condições experimentais. À medida que o tempo vai aumentando essa concentração diminui e atingi um mínimo de 0,00336 mol $\mathrm{L}^{-1}$ para as três condições, indicando o ponto de saturação. As concentrações de $\mathrm{CO}_{2}$ iniciam-se diferentes entre os três experimentos, elas aumentaram com o tempo atingindo valores semelhantes entre si com $0,06467 \mathrm{~mol} \mathrm{~L}^{-1}, 0,06887 \mathrm{~mol} \mathrm{~L}^{-1}$ e $0,07140 \mathrm{~mol} \mathrm{~L}^{-1}$ para as vazões de 
20, 10 e $5 \mathrm{~L} \mathrm{~min}^{-1}$ de $\mathrm{CO}_{2}$ respectivamente.

Uma possível justificativa para a menor absorção de $\mathrm{CO}_{2}$ na solução na primeira condição é que a vazão de $\mathrm{CO}_{2}$ foi maior que a vazão de ar. Em contrapartida, uma vazão mais reduzida, como é o caso da terceira condição, acarreta na necessidade de mais tempo para atingir o máximo de absorção de $\mathrm{CO}_{2}$.

As variações das concentrações de $\mathrm{CO}_{2}$ com o tempo ficam com melhor visualização na Figura 1.

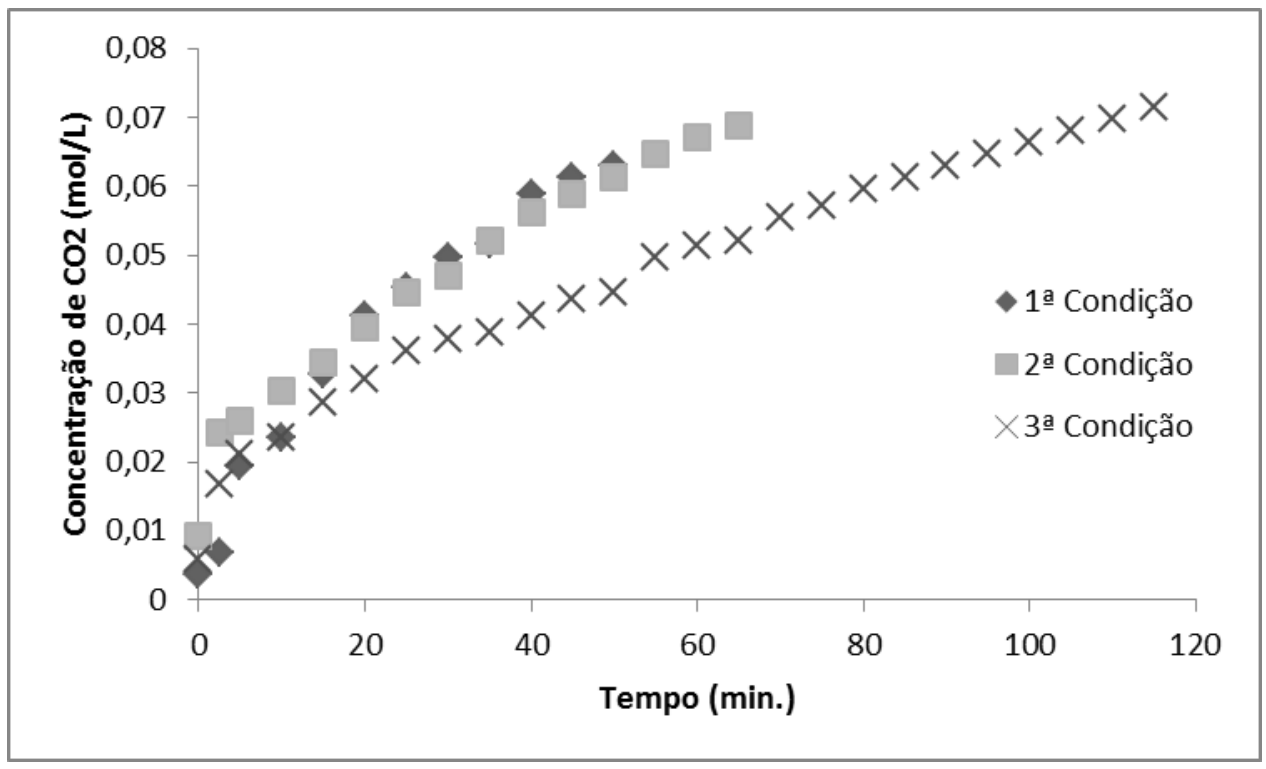

Figura 1 - Absorção de $\mathrm{CO}_{2}\left(\mathrm{~mol} \mathrm{~L}^{-1}\right)$ em função do tempo

A Figura 1 mostra que a relação de absorção com o tempo não é linear. A primeira condição, com alimentação de $\mathrm{CO}_{2}$ de $20 \mathrm{~L} \mathrm{~min}^{-1}$ atinge a concentração máxima de absorção em 55 minutos. Apesar de a segunda condição possuir a metade da vazão de alimentação de $\mathrm{CO}_{2}(10$ $\mathrm{L} \min ^{-1}$ ) o tempo gasto para atingir a saturação de absorção foi apenas 10 minutos a mais que a primeira (65 min). Porém essa relação com o tempo não é proporcional, pois para a alimentação de $5 \mathrm{~L} \mathrm{~min}^{-1}$ de $\mathrm{CO}_{2}$ o tempo para absorção máxima foi de 115 minutos.

A relação percentual entre o número de mols de $\mathrm{CO}_{2}$ absorvido com o número de mols de $\mathrm{NaOH}$ na solução pode ser expressa pela razão entre o número de mols do componente absorvente $(\mathrm{NaOH})$ e o número de mols de absorvido $\left(\mathrm{CO}_{2}\right)$ multiplicada por $100 \%$

A porcentagem é mais bem visualizada na forma de gráfico como apresentado na Figura 2. 


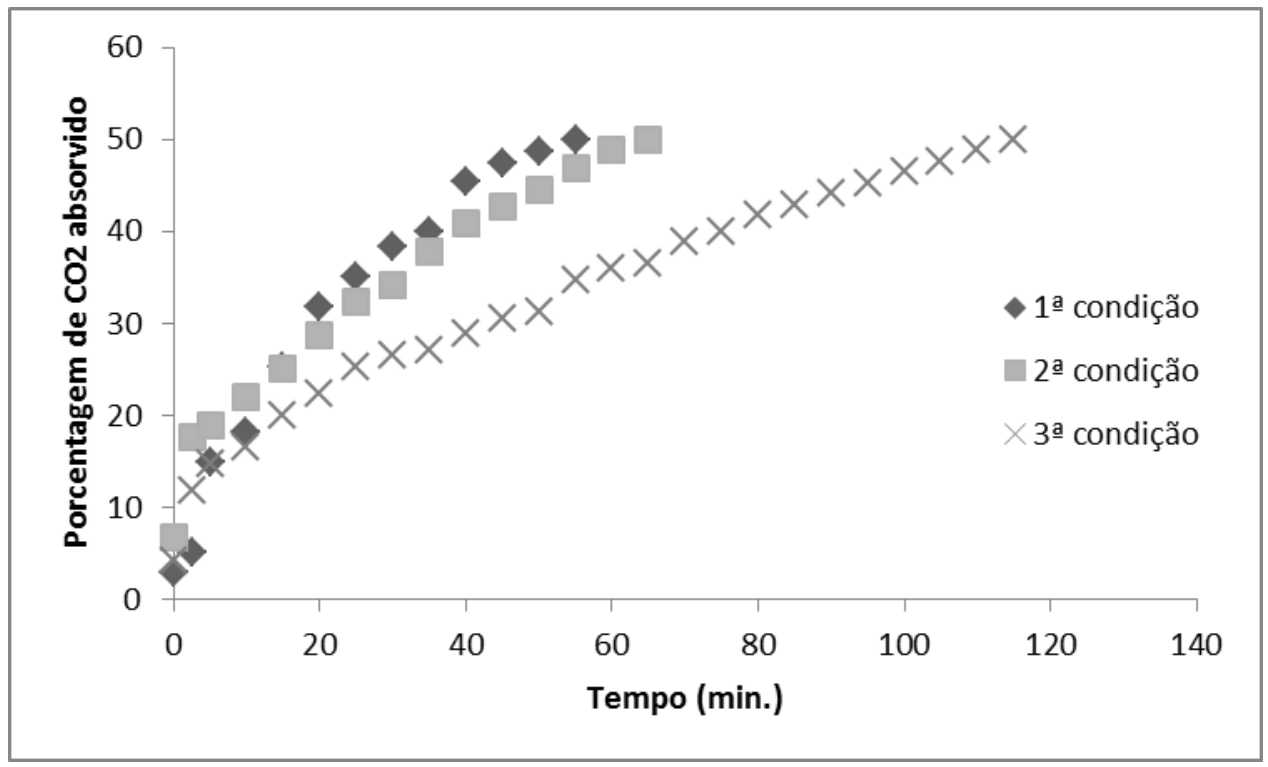

Figura 2 - Gráfico da porcentagem de $\mathrm{CO}_{2}$ absorvido, com relação à $\mathrm{NaOH}$, pelo tempo.

A porcentagem de $\mathrm{CO}_{2}$ desse gráfico considera a quantidade de $\mathrm{NaOH}$ reagido na solução absorvente. Pelo fato das três condições possuírem concentrações de hidróxido de sódio similares entre si, as porcentagens finais de absorção também devem ser próximas. O que varia neste caso é o tempo para atingir o valor máximo de absorção. A diferença de porcentagem foi ínfima podendo arredondar as três porcentagens em 50\%. As máximas absorções de $\mathrm{CO}_{2}(50 \%)$ foram atingidas na primeira, segunda e terceira condições após 55, 65 e 115 minutos de processo. Esses valores já eram esperados uma vez que a relação estequiométrica da reação é de 2 mols de $\mathrm{NaOH}$ para 1 mol de $\mathrm{CO}_{2}$

Outro fator que também pode ser analisado é a porcentagem de recuperação. Ela considera a quantidade de $\mathrm{CO}_{2}$ que é recuperado em relação à fase gasosa da coluna. Essa porcentagem é calculada a partir das frações de $\mathrm{CO}_{2}$ por ar sem $\mathrm{CO}_{2}$ na base e no topo da coluna. A Figura 3 mostra a recuperação de $\mathrm{CO}_{2}$ com o tempo para as três condições estudadas. 


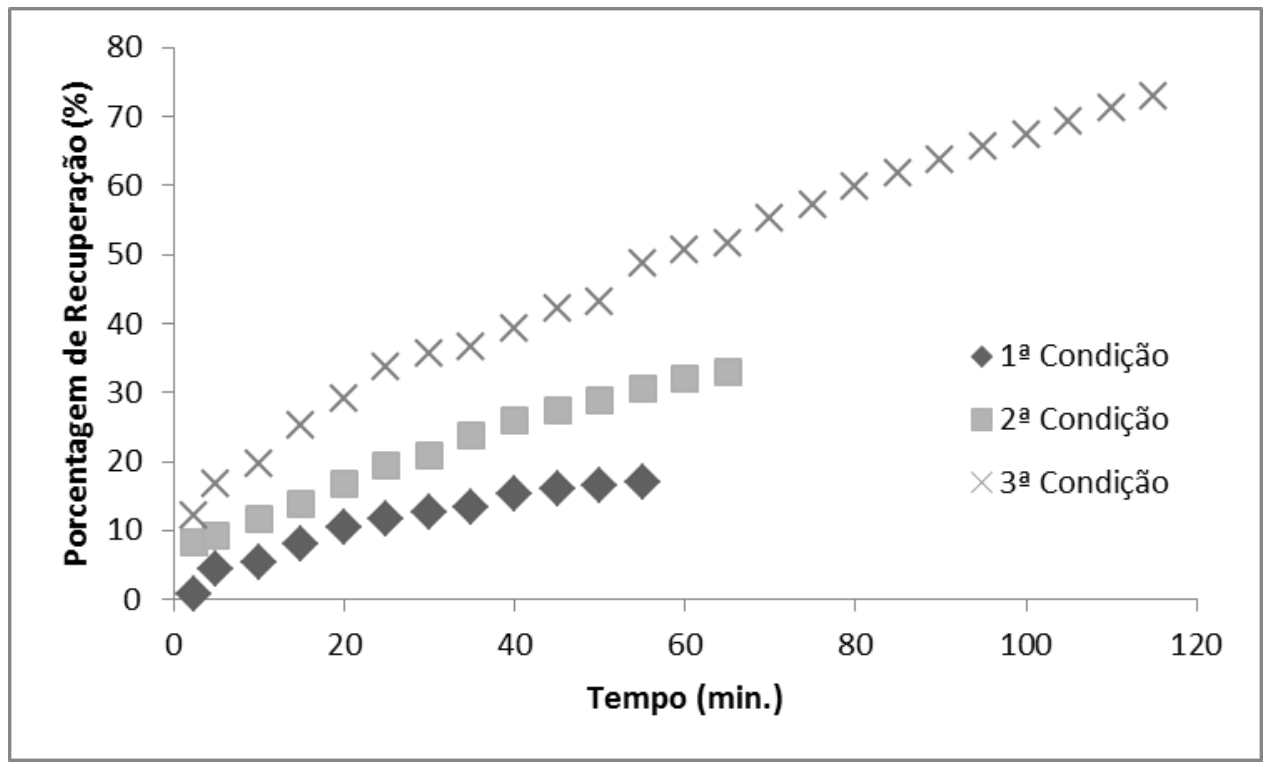

Figura 3 - Porcentagem de recuperação de $\mathrm{CO}_{2}$ com o tempo.

A porcentagem de recuperação de $\mathrm{CO}_{2}$ na primeira condição foi de $16,90 \%$, na segunda condição $32,92 \%$ e na terceira condição $73,00 \%$. Essas porcentagens são em relação à quantidade de $\mathrm{CO}_{2}$ na vazão gasosa. Como no final as concentrações de hidróxido de sódio, nas três condições são iguais, essa recuperação deve ser maior para o gás pobre em $\mathrm{CO}_{2}$. $\mathrm{Ou}$ seja, quanto menor a vazão volumétrica de $\mathrm{CO}_{2}$ no gás, maior será a porcentagem recuperada de $\mathrm{CO}_{2}$.

Segundo Yoo et al. (2013), a taxa de absorção é proporcional a concentração de $\mathrm{NaOH}$. Portanto, o enriquecimento da solução de $\mathrm{NaOH}$ torna a absorção de $\mathrm{CO}_{2}$ mais eficaz em detrimento a variação de vazão do gás.

Outro parâmetro de grande importância na análise de absorção de gases é o coeficiente de transferência de massa. A determinação desse coeficiente é dada pela Equação 1.

$$
\mathrm{k}_{\mathrm{y}}^{\mathrm{a}}=\frac{\mathrm{v}}{\mathrm{H}_{\mathrm{OG}} \mathrm{A}}
$$

Sendo $\mathrm{V}$ a vazão molar do gás inerte (ar), $\mathrm{H}_{\mathrm{OG}}$ a altura de uma unidade de transferência e A a área de seção transversal da coluna.

Os resultados encontrados para o coeficiente global de transferência de massa $\left(\mathrm{k}_{\mathrm{y}} \mathrm{a}\right)$ foram:

$1^{\mathrm{a}}$ condição $\left(20 \mathrm{~L} \mathrm{~min}^{-1}\right.$ de $\left.\mathrm{CO}_{2}\right)-0,04242 \mathrm{~mol} \mathrm{~min}^{-1} \mathrm{~m}^{-3}$;

$2^{\mathrm{a}}$ condição $\left(10 \mathrm{~L} \mathrm{~min}^{-1}\right.$ de $\left.\mathrm{CO}_{2}\right)-0,07190 \mathrm{~mol} \mathrm{~min}^{-1} \mathrm{~m}^{-3}$;

$3^{\mathrm{a}}$ condição $\left(5 \mathrm{~L} \mathrm{~min}^{-1}\right.$ de $\left.\mathrm{CO}_{2}\right)-0,08690 \mathrm{~mol} \mathrm{~min}^{-1} \mathrm{~m}^{-3}$. 


\section{9 a 22 de outubro de 2014 \\ Florianópolis/SC}

Como pode ser observado, os coeficientes de transferência de massa e a porcentagem de recuperação foram inversamente proporcionais à vazão de $\mathrm{CO}_{2}$ utilizada. Ou seja, quando foi empregada uma vazão de $\mathrm{CO}_{2}$ de $20,0 \mathrm{~L} \mathrm{~min}{ }^{-1}$, que é o valor mais alto utilizado nesse experimento, obteve-se o menor coeficiente de transferência de massa de $0,04242 \mathrm{~mol} \mathrm{~min}^{-1} \mathrm{~m}^{-3} \mathrm{e}$ a menor porcentagem de recuperação de $\mathrm{CO}_{2}$ de $16,90 \%$. Em contrapartida, para a vazão mais baixa de $\mathrm{CO}_{2}$, de $5 \mathrm{~L} \mathrm{~min}^{-1}$, alcançou-se o maior coeficiente de transferência de massa, 0,0869 mol $\min ^{-1} \mathrm{~m}^{-3}$, e a maior porcentagem de recuperação do referido gás, $73,00 \%$.

Os resultados de $\mathrm{k}_{\mathrm{y} a}$ são de grande importância, pois os valores instantâneos de transferência de massa são correspondentes aos pontos na coluna e podem variar consideravelmente da entrada até a saída da coluna, sendo assim o kya que é o coeficiente global fornece a média necessária para uma melhor análise. É importante perceber que a absorção é um fenômeno físico complexo e que o coeficiente $\mathrm{k}_{\mathrm{y}} \mathrm{a}$ é mais uma variável de correlação empírica do que uma taxa de velocidade exata. Este coeficiente por si só pode ter pouco significado prático, uma vez que altos valores não indicam, necessariamente, elevada absorção (Houghton, 1956).

No modelamento dos efeitos da alimentação de $\mathrm{CO}_{2}$ sobre a área efetiva de transferência de massa (a), desenvolvido por Cormos et al. (2012) foi observado que quanto maior for a velocidade deste gás na coluna de absorção, menor será a área de transferência de massa efetiva. Convergindo com os resultados deste trabalho, no sentido de inferir que vazões menores de $\mathrm{CO}_{2}$ propiciam uma transferência de massa mais efetiva entre este gás e o absorvente utilizado, $\mathrm{NaOH}$, o que implica em recuperação mais elevada de $\mathrm{CO}_{2}$ nesta condição.

A quantificação das características de transferência de massa na absorção é de difícil determinação. Isto resultou na publicação de Valens et al.(2009) que em seu trabalho comparou resultados de diversos autores e concluiu que há muita divergência nos valores encontrados para o coeficiente global de transferência de massa na absorção de $\mathrm{CO}_{2}$ em $\mathrm{NaOH}$. Portanto, comparar valores de kya não é um bom argumento de análise. Valens et al. (2009) no entanto não invalidou a relação de maiores coeficiente de transferências de massa para menores vazões de $\mathrm{CO}_{2}$. Na prática esses valores conflitantes para esse parâmetro são fruto da grande quantidade de dados que são avaliados por diferentes métodos. O que força os engenheiros a superestimar grandemente parâmetros de coluna em seus projetos (Valens et al., 2009).

\section{CONCLUSÃO}

Este estudo mostra que o hidróxido de sódio é um bom absorvente de $\mathrm{CO}_{2}$ e que cálculos estequiométricos são capazes de fornecer dados fundamentais para a análise da absorção. Quando se trata do número de mols de $\mathrm{CO}_{2}$ absorvido em relação ao hidróxido de sódio inicial tem-se que quanto maior a vazão de $\mathrm{CO}_{2}$ mais rápido se atinge a saturação da solução de $\mathrm{NaOH}$. Para as condições estudadas a saturação foi de praticamente $50 \%$ da massa de $\mathrm{NaOH}$ inicial. Já a porcentagem de recuperação relaciona a quantidade de $\mathrm{CO}_{2}$ na vazão gasosa, quanto menor a vazão volumétrica de $\mathrm{CO}_{2}$ no gás maior será a porcentagem recuperada. Portanto, a porcentagem de recuperação máxima foi de $73 \%$ para a menor vazão de $\mathrm{CO}_{2}: 5 \mathrm{~L} \mathrm{~min}^{-1}$. A mesma relação inversa ocorre para o coeficiente de transferência de massa. A menor vazão de $\mathrm{CO}_{2}, 5 \mathrm{~L} \mathrm{~min}^{-1}$, 
possui o maior coeficiente $\mathrm{k}_{\mathrm{y}}$ a $0,08690 \mathrm{~mol} \mathrm{~min}^{-1} \mathrm{~m}^{-3}$, ou seja, houve uma variação do coeficiente de transferência de massa e da porcentagem de recuperação inversamente proporcional às vazões de $\mathrm{CO}_{2}$ adotadas no experimento.

\section{REFERÊNCIAS}

AROONWILAS, Adisorn et al. Mathematical modelling of mass-transfer and hydrodynamics in $\mathrm{CO} 2$ absorbers packed with structured packings. Chemical Engineering Science, Ontario, Canada, v. 58, p.4037-4053, 2003.

CORMOS, A. M; GASPAR, J. Assessment of mass transfer and hydraulic aspects of CO2 absorption in packed columns. International Journal of Greenhouse Gas Control, Cluj-Napoca, Romania, v. 6, p. 201-209, 2012.

HOUGHTON, G. et al. Absorption of carbon dioxide in water under pressure using a gas-bubble column. Chemical Engineering Science, Glasgow, v. 7, p.26-39, 1956.

VALENZ, L. et al. Methods standardization in the measurement of mass-transfer characteristics in packed absorption columns. Chemical Engineering Research And Design, Prague, Czech Republic, v. 87, p.695-704, 2009.

YOO, M.; HAN, S., WEE, J.Carbon dioxide capture capacity of sodium hydroxide aqueous solution. Journal of Environmental Management, Gyeonggi-do, Republic of Korea, v.114 p. 512-519, 2013. 